\title{
Optimal allocation of limited resources among discrete risk-reduction options
}

\author{
Michael Todorov Todinov* \\ Department of Mechanical Engineering and Mathematical Sciences, Oxford Brookes University, United Kingdom
}

Received: August 4, 2014

Accepted: September 3, 2014 Online Published: September 17, 2014

DOI: $10.5430 /$ air.v3n4p15

URL: http://dx.doi.org/10.5430/air.v3n4p15

\begin{abstract}
This study exposes a critical weakness of the (0-1) knapsack dynamic programming approach, widely used for optimal allocation of resources. The (0-1) knapsack dynamic programming approach could waste resources on insignificant improvements and prevent the more efficient use of the resources to achieve maximum benefit. Despite the numerous extensive studies, this critical shortcoming of the classical formulation has been overlooked. The main reason is that the standard (0-1) knapsack dynamic programming approach has been devised to maximise the benefit derived from items filling a space with no intrinsic value. While this is an appropriate formulation for packing and cargo loading problems, in applications involving capital budgeting, this formulation is deeply flawed. The reason is that budgets do have intrinsic value and their efficient utilisation is just as important as the maximisation of the benefit derived from the budget allocation.

Accordingly, a new formulation of the (0-1) knapsack resource allocation model is proposed where the weighted sum of the benefit and the remaining budget is maximised instead of the total benefit. The proposed optimisation model produces solutions superior to both - the standard (0-1) dynamic programming approach and the cost-benefit approach.

On the basis of common parallel-series systems, the paper also demonstrates that because of synergistic effects, sets including the same number of identical options could remove different amount of total risk. The existence of synergistic effects does not permit the application of the (0-1) dynamic programming approach. In this case, specific methods for optimal resource allocation should be applied. Accordingly, the paper formulates and proves a theorem stating that the maximum amount of removed total risk from operations and systems with parallel-series logical arrangement is achieved by using preferentially the available budget on improving the reliability of operations/components belonging to the same parallel branch. Improving the reliability of randomly selected operations/components not forming a parallel branch leads to a sub-optimal risk reduction. The theorem is a solid basis for achieving a significant risk reduction for systems and processes with parallel-series logical arrangement.
\end{abstract}

Key Words: Risk reduction, (0-1) Knapsack dynamic programming, Cost-benefit analysis, Parallel-series arrangement

\section{Introduction and related work}

The problem of optimal resource allocation to attain a maximum benefit is an important problem, which appears frequently in capital budgeting of departments and companies, cutting stock problems, packing and cargo loading problems.

One of the most commonly used methods for solving the discrete resource allocation problem is the classical $0-1$

\footnotetext{
*Correspondence: Michael Todorov Todinov; Email: mtodinov@brookes.ac.uk; Address: Department of Mechanical Engineering and Mathematical Sciences, Oxford Brookes University, United Kingdom 
knapsack dynamic programming approach which is also one of the most extensively studied combinatorial optimization problems. According to the classical formulation of this problem, a set $\Omega$ of possible independent options are given, each of which brings benefit of magnitude $r_{i}(i=$ $1,2, \cdots, n)$. The individual options $i$, are also characterised by their implementation costs $c_{i}$. The options can be selected only once, hence each option can either be accepted or rejected. No option can be repeated and no option can be split. The task of optimal allocation of the available budget consists of determining the optimal subset $P \subseteq \Omega$ of options associated with the maximum total benefit $\sum_{k \in P} r_{k}$. The imposed constraint is the specified limited budget $B$ : the total cost of the selected $n$ options must not exceed the available budget $B$ :

$$
\text { Maximize : } \sum_{i=1}^{n} x_{i} \times r_{i}
$$

subject to the constraint:

$$
\sum_{i=1}^{n} x_{i} \times c_{i} \leq B
$$

where $x_{i} \in\{0,1\}$ are decision variables; $x_{i}=1$ if the riskreduction option is accepted and $x_{i}=0$, otherwise. Normally, the sum of the costs of all options exceeds the existing budget $B\left(\sum_{k \in P} c_{k}>B\right)$. The implicit assumption behind this formulation is that each option can be independently implemented with no consequences to other options. ${ }^{[1]}$

From (1)-(2) it is clear, that the purpose of the classical formulation of the knapsack problem is to maximise the value derived from filling an empty 'knapsack' of given size B, with no intrinsic value. For optimal packing and cargo loading problems this formulation is entirely adequate because the space filled with items has no intrinsic value. For resource allocation problems however, the available budget for achieving the desired maximum benefit, does have intrinsic value. The efficient use of the budget is just as important as the benefit derived from its allocation.

The (0-1) knapsack dynamic programming approach has been intensively studied and used for a long time for optimal allocation of resources, ${ }^{[2-9]}$ and in particular, as a resource allocation method among competing projects. More recently, the 0-1 knapsack problem has also been used and discussed. ${ }^{[11-16]}$

No discussion however seems to exist on the possibility of obtaining inferior solutions from the classical (0-1) knapsack problem formulation (1)-(2) compared to the competing cost-benefit approach for optimal allocation of resources. Despite the very large volume of existing research related to the (0-1) knapsack dynamic programming approach, this important point has been overlooked.
Furthermore, despite that dynamic programming techniques have been around for a long time, ${ }^{[17]}$ very few attempts have been made to use them for optimal allocation of riskreduction resources. Richter et al. ${ }^{[18]}$ solved an optimal resource allocation problem to achieve a maximum prevention from infection. The objective function of the formulated model involved only two additive terms, corresponding to two independent populations. No details however were provided about the optimization algorithm.

A dynamic programming solution of the safety resource allocation problem, in the case where the functions describing the risk reduction are arbitrary continuous functions, has been presented in Todinov. ${ }^{[19]}$ The treatment however, did not cover the common case of discrete risk-reduction options. Mehr and Tumer ${ }^{[20]}$ solved the optimal budget allocation problem as a portfolio optimization problem, similar to the problem commonly solved in managing investment portfolios. However, this model has a narrow application and cannot be used in the common case of discrete risk reduction options.

Indeed, the risk is often reduced by well-defined discrete options: purchasing new, more reliable and safer equipment, investing in personnel training, investing in improved security and control, investing in new systems, etc. Each riskreduction option can either be accepted (included) in the optimal set of options or not. For each risk-reduction option, it is usually known from statistical data and experience, how large risk-reduction effect is achieved from implementing the option. For example, in the railway industry, the risk-reduction effect is commonly measured by estimating the expected number of prevented fatalities and injuries from implementing a particular option. ${ }^{[21]}$ In the case of discrete risk-reduction options, cost benefit analysis has been adopted by many industries and in particular by the railway industry, as a tool for optimal allocation of safety resources. In the railway industry for example, the safety budget allocation starts with assigning risk reduction options to the different risk contributors or risk scenarios resulting in a major railway accident. Each risk reduction option is assessed in terms of the benefit it brings and the cost of its implementation. The risk reduction options are ranked according to their benefit/cost ratio. By starting with the risk reduction option with the largest benefit/cost ratio, the options are sequentially included in the optimal set and a check is performed whether the aggregated cost of the selected risk reduction options has exceeded the allocated budget. The risk-reduction options whose aggregated cost is within the allocated budget are included in the optimal set. Consequently, the algorithm of the cost-benefit approach can be described by the following basic steps: ${ }^{[21]}$

(1) Rank the risk-reduction options in descending order, according to their benifit/cost ratio.

(2) Choose the risk-reduction option, with the highest benifit/cost ratio, which fits in the remaining budget. 
(3) Update the remaining budget.

(4) Repeat steps 2 and 3 until no other risk-reduction option can be included in the optimal set.

For $n$ risk-reduction options, the ranking in descending order, according to the benefit/cost ratio, can be done in $O(n \log n)$ time. As a result, the selection of risk-reduction options by following the cost-benefit method can always be made in $O(n \log n)$ time.

There have been attempts to substitute the cost-benefit analysis selection of discrete risk reduction options with the (0-1) knapsack dynamic programming approach. Thus, Pigman et $\mathrm{al}^{[22]}$ developed a dynamic procedure based on the (0-1) knapsack dynamic programming approach which selects the optimal combination of safety improvement projects for a given budget. Pigman et $\mathrm{al}^{[22]}$ compared the (0-1) dynamic programming method with the cost-benefit approach and concluded that because the (0-1) knapsack programming procedure is not constrained by the benefitcost ratios, it utilises better the available resources and is a superior method to the cost-benefit analysis.

More recently, the classical (0-1) knapsack dynamic programming method in combination with the risk matrix has been advocated for optimal risk reduction in Ref. 1. All risks have been classified in the cells of a $4 \times 5$ risk matrix and investments are made to decrease the risk in cell $i$ towards a cell $j$ with a smaller risk. However, no details about the implementation algorithm have been presented.

A detailed (0-1) knapsack dynamic programming algorithm for optimal allocation of risk-reduction resources in the case of discrete and statistically independent risk-reduction options was described in Ref. 23. Central to this algorithm was the concept 'amount of removed risk' (expected potential loss) characterising the individual options and measuring the derived benefit from their application. The removed risk was expressed in monetary terms - the expected cost of prevented accidents and fatalities.

The individual risk-reduction options $i(i=1,2, \cdots, n)$ are characterised by the amount of removed risk $r_{i}$ (expected potential loss) and cost of implementation $c_{i}$. Risk reduction options can be selected only once, hence each risk reduction option can either be accepted or rejected.

The risk-reduction problem solved in Ref. 23 followed the classical formulation of the (0-1) knapsack dynamic programming (1)-(2). This choice was justified by the nature of the considered problem: optimal allocation of a cental safety budget for removing as many expected human fatalities as possible. In this case, the purpose is not to save resources and transfer them elsewhere, but to use the available budget to the full and prevent as many expected fatalities as possible.

In cases concerned with preventing financial losses or in cases where unused budgets can be transferred for risk reduction in other areas, the unused budget is just as valuable as the derived benefit from the invested part of the budget. In these common cases, recent studies exposed an unexpected weakness of the classical (0-1) knapsack dynamic programming formulation (1)-(2). The solutions based on the classical knapsack dynamic programming formulation (1)-(2) could waste resources on insignificant increase of the derived benefit.

In addition, the presence of independent risk-reduction options whose implementation does not affect other options, does not guarantee that the (0-1) dynamic programming approach could be applied. The existence of synergistic effects related to the selected sets of options does not permit the application of the (0-1) dynamic programming approach. In this case, other methods for optimal resource allocation should be used.

\section{The proposed method}

\subsection{A counter-example}

From the analyses published in the literature so far, it seems that the standard (0-1) knapsack approach is a real alternative to the cost-benefit approach. This perception however is rather deceptive as the next counter-example reveals. Suppose that the benefits and the costs of four risk reduction options preventing potential warranty costs are according to Table 1 . The available safety budget is 30 million.

Table 1: Four risk reduction options each characterised with cost of implementation and magnitude of the removed risk. The total safety budget is 30 million.

\begin{tabular}{llll}
\hline $\begin{array}{l}\text { Risk-reduction } \\
\text { option }\end{array}$ & $\begin{array}{l}\text { Removed risk } \\
\text { [in millions \$] }\end{array}$ & $\begin{array}{l}\text { Cost of implementation } \\
\text { [in millions \$] }\end{array}$ & $\begin{array}{l}\text { Benefit/Cost } \\
\text { ratio }\end{array}$ \\
\hline A & 33 & 10 & 3.3 \\
B & 20.9 & 7 & 2.98 \\
C & 26 & 14 & 1.86 \\
D & 28 & 16 & 1.75 \\
\hline
\end{tabular}

All risk reduction options are characterised by a benefit/cost ratio greater than one. The standard (0-1) knapsack algorithm, selects risk reduction options $\mathrm{C}$ and $\mathrm{D}$, which, within the fixed budget of 30 million, yield the largest risk reduction (54 million). Clearly, this is a flawed solution because if risk reduction options $A$ and $B$ had been selected, the risk reduction would be marginally smaller (53.9 million) but 13 million unnecessary expenses (43\% of the budget) would have been saved. In fact, the classical (0-1) knapsack algorithm 'chooses' to spend 13 million towards a risk reduction of only 0.1 million, which effectively has a benefit/cost ratio $0.1 / 13=0.0077$. This is an indication of an extremely wasteful use of resources!

It needs to be pointed out that in this counter-example, the cost-benefit approach selects correctly the risk reduction op- 
tions $\mathrm{A}$ and $\mathrm{B}$ and avoids the problem associated with the standard (0-1) knapsack approach.

Suppose that $T R_{(0-1)}$ and $T C_{(0-1)}$ denote the total removed risk and the total cost of the selected options characterising the (0-1) classical knapsack dynamic programming solution, respectively. Similarly, $T R_{(C B)}$ and $T C_{(C B)}$ denote the total reduced risk and the total cost of the selected risk reduction options characterising the cost-benefit solution. The comparative risk-reduction effectiveness ratio $\rho$ can then be calculated from

$\rho=\left(T R_{(0-1)}-T R_{(C B)}\right) /\left|T C_{(0-1)}-T C_{(C B)}\right|$

This ratio measures the effectiveness of the extra budget used by the standard (0-1) knapsack algorithm for reducing risk, compared to the cost-benefit method.

If the risk-reduction ratio $\rho$ is too small, the classical (01) dynamic programming solution achieves only a marginal risk reduction, at a very large cost, and should be discarded in favour of the cost-benefit analysis solution. If the riskreduction ratio $\rho$ indicates that a substantial risk reduction has been achieved with the extra budget, the (0-1) knapsack solution results in a cost-effective risk reduction and should be accepted as an alternative of the cost-benefit solution.

From Table 1, $T R_{(0-1)}=26+28=54 ; T C_{(C B)}=$ $33+20.9=53.9 ; T C_{(0-1)}=16+14=30$ and $T C_{(C B)}=$ $10+7=17$. The comparative ratio given by equation (2) then becomes: $\rho=(54-53.9) /|30-17|=0.0077$

which is only $0.7 \%$. This ratio indicates a very inefficient use of safety resources and the standard (0-1) knapsack solution is worse than the cost-benefit solution.

The main reason for this problem is that the classical (0-1) knapsack approach has actually been devised to maximise the total benefit derived from items filling space with no intrinsic value.

It needs to be pointed out that if the budget had no intrinsic value, then the (0-1) dynamic programming approach would always yield an optimal solution. The budget however, does have intrinsic value and is just as important as maximising the risk reduction, particularly in the common case of risk reduction related to financial losses.

The direct application of the (0-1) dynamic programming approach devoid from the intrinsic value of the budget leads to resources being trapped on insignificant improvements and prevents their efficient use.

For the separate departments in a company for example, it is important to determine how to allocate their limited safety resources in order to mitigate a number of relevant sources of risk. A fixed budget constraint is always present if the total cost of the available risk-reduction options is greater than the amount of available resources.

Wastage of resources on an insignificant improvement exists even in situations where the purpose of the risk reduction budget is the prevention of as many human fatalities as possible. Consider an example related to preventing accidents in the railway industry. Suppose that safety budgets are allocated for improving the safety of level crossings, reducing the risk of derailment, reducing the risk of train collision and reducing the train platform accidents. Each of these areas of safety improvement is associated with its distinct set of possible risk reduction options. In each of these areas, it is required to identify the optimal selection of options which achieves the most efficient risk reduction within the allocated resources for that particular area. It is very important not to waste resources on insignificant risk reduction in any of these areas because remaining (unused) resources in any of these areas could be re-directed to prevent fatalities in the other areas. In this sense, the more efficient use of risk reduction resources in each area ultimately translates into a higher total number of prevented fatalities.

A very similar situation is present in the cases where safety budges are allocated for reducing the risk of certain diseases. Each particular disease is associated with its own distinct set of possible risk reduction options. Again, it is important not to waste resources on insignificant risk reduction in any of these risk areas because remaining resources in one of these areas could be transferred for more efficient disease prevention in the other areas. If resources are wasted on an insignificant reduction of the expected fatalities from diseases $\mathrm{A}, \mathrm{B}$ and $\mathrm{C}$ for example, there may not be resources left for preventing significantly more expected fatalities from the equally dangerous disease $\mathrm{D}$. The result is an inefficient use of resources.

Unfortunately, often it is impractical to state and solve the budget allocation problem as a global optimisation problem at the level of the entire organisation (company), including all possible risk reduction options across all departments.

The first reason is technical-the dimension of the problem is very big. The presence of thousands of risk reduction options makes it extremely difficult to apply an exact optimisation technique such as dynamic programming. In addition, it is difficult to provide consistency and coordination across many departments. As a result, some of the selected risk reduction options will be incompatible or may require complex conditions and constraints which could be difficult to resolve on a global scale.

The second reason is that deciding upon and listing all available risk reduction options in a particular area, and not missing any risk reduction option, can only be done by experts with deep knowledge relevant to that particular area. As a result, the problem of optimal allocation of resources is a problem that needs to be solved by each responsible department.

Finally, decomposing the problem of global optimisation into series of smaller-size problems solved by the relevant 
departments is essentially an application of the 'divide and concur strategy' which has already proved its benefits in solving efficiently large-scale optimisation problems.

\subsection{Synergistic effects associated with independent risk reduction options}

The implicit assumption behind the classical (0-1) knapsack allocation problem formulation (1-2) is that the separate options are independent and their individual implementation does not affect other options. However, the next example shows that even if this assumption is fulfilled, in some cases, the (0-1) knapsack allocation still cannot be applied because of the synergistic effect from particular option selections.

Consider the system in Figure 1 which transports water from three sources $\mathrm{s} 1, \mathrm{~s} 2$ and $\mathrm{s} 3$ to a processing plant $t$. The water supply system consists of identical pipeline sections (the arrows in Figure 1a). The pipeline sections are subjected to random ruptures caused by intensive corrosion. The places of intensive corrosion are randomly located and it can be considered that each section essentially fails independently from the other sections. In addition, the replacement of any section has no effect on other sections. In other words, the separate pipeline sections can be regarded as independent components.

Suppose that the water supply system fulfils its mission only if at least one parallel branch delivers water to the processing plant. As a result, a system failure is defined by a state for which none of the parallel branches delivers water to the processing plant.

Suppose that each pipeline section is characterized by the same probability (for example 0.6) of working after one year of continuous operation. Because of the deteriorated pipeline sections, the water supply system will certainly benefit from risk-reduction options consisting of purchasing and replacing old (deteriorated) pipeline sections (marked with 'o' in Figure 1) with new sections (marked with ' $n$ ' in Figure 1). Consequently, the replacement of any of the nine independently working pipeline sections can be viewed as a possible risk-reduction option. Now suppose that the available budget is sufficient for implementing exactly three options (for replacing exactly three pipeline sections). Suppose also that each new pipeline section is characterised by a probability 0.95 of working after one year of continuous operation.

The identical pipeline sections are independent and interchangeable and the replacement of any section has no effect on the other sections. In addition, the selection of any three sections for a replacement results in the same expected number of prevented component failures in the system. Because of the symmetry of the system in Figure 1a, it seems that any three risk-reduction options could be replaced with new sec- tions (see Figure 1b, Figure 1c and Figure 1d), to the same effect. This conclusion however would be incorrect.

Because of the synergistic effect of the selected options, the total removed risk is highest if the available budget is spent preferentially on replacing pipeline sections forming an entire parallel branch (see Figure 1d), as opposed to replacing randomly selected sections from different branches (see Figure 1b,Figure 1c). a)

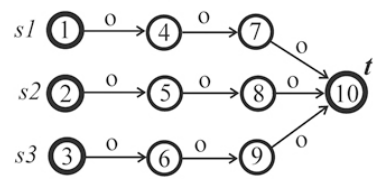

c)

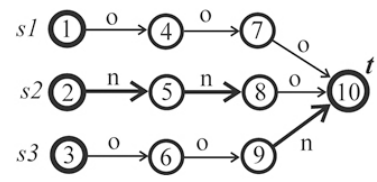

b)

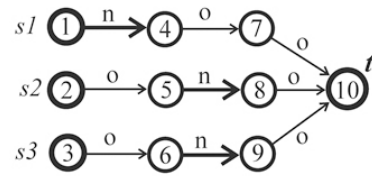

d)

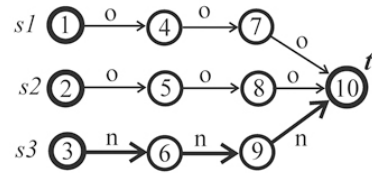

Figure 1: A water supply system consisting of three parallel branches including three separate sections.

Indeed, the probability that there will be at least one branch delivering water after one year of operation, if one section is replaced from each parallel branch (see Figure 1b), is:

$R_{b}=1-\left(1-0.6^{2} \times 0.95\right)^{3}=0.71$

The probability that there will be at least one branch delivering water after one year of operation, if two sections are replaced from the same branch, and one section from another branch (see Figure 1c) is:

$R_{c}=1-\left(1-0.6^{3}\right) \times\left(1-0.95^{2} \times 0.6\right) \times\left(1-0.6^{2} \times 0.95\right)=$ 0.76

The probability that there will be at least one branch delivering water after one year of operation, if three sections from the same branch are replaced is:

$R_{d}=1-\left(1-0.6^{3}\right)^{2} \times\left(1-0.95^{3}\right)=0.91$

This example shows that in some cases, even for identical and independent risk-reduction options whose individual implementation has no consequences for other options, the amount of total removed risk depends on the actual selection of the risk reduction options.

Consider now the case where the water supply system fulfils its mission, only if all three branches deliver water to the processing plant. If no old sections are replaced with new ones, the probability of no failure of the water supply within one year of operation is

$R=0.6^{9}=0.01$

which is the probability that all nine sections will be in 
working state at the end of the year.

If exactly three sections are replaced, no matter which three sections are replaced, the probability of no disruption in the water supply, within one year of operation, remains the same:

$R=0.6^{6} \times 0.95^{3}=0.04$

In other words, in this case, the same amount of total risk is removed, irrespective of the selected set of risk reduction options. This different result, obtained for the same physical system, can be explained by the fact that despite that the nine water sections are physically still arranged as a parallelseries system, with respect to delivering a full-capacity flow of water to the processing plant, they are logically arranged in series. In this case, the synergistic effect from the option selection is not present and the total amount of removed risk is insensitive to which set of three options is selected.

The considered example involving a parallel-series logical arrangement is very common. It holds for example in the case where the risk-reduction options are investments in training people.

Indeed, consider the case where three groups of people (teams) A, B and C, each of which includes three independently working team members, work towards eliminating the same major hazard (see Figure 2). The risk is reduced if at least one of the teams succeeds in eliminating the hazard. Within each team, the task of eliminating the major hazard is divided into subtasks among the team members. Every single person in a team must accomplish their sub-task successfully, in order for the team to eliminate the hazard successfully.

The identical risk reduction options are the resources invested in training a single person. The training increases the probability that the sub-task conducted by the person will be accomplished successfully. Suppose that an untrained person accomplishes successfully their sub-task with probability $k(0 \leq k<1)$, while investing in training of that person increases the probability of accomplishing the task to $m>k(m<1)$.

If the available budget is sufficient to train three people only, a random selection of three people for training, from different groups is a strategy far from optimal. It results in probability

$p_{a}=1-\left(1-k^{2} m\right)^{3}$

of removing the hazard for the case in Figure $2 \mathrm{a}$ and probability

$p_{b}=1-\left(1-k^{3}\right)\left(1-k m^{2}\right)\left(1-k^{2} m\right)$

for the case in Figure 2b.

Investing in training all the people from a single team results in the highest probability $p_{c}=1-\left(1-k^{3}\right)^{2}\left(1-m^{3}\right)$

of successfully accomplishing the task.

Indeed, and the difference $p_{c}-p_{a}$ can be presented as

$p_{c}-p_{a}=(k-m)^{2}\left[2 k\left(1-m k^{2}\right)+m-k^{4}\right]$

In this expression, $m<1$ and $k<1$, hence $1-m k^{2}>0$. In addition, because $m<1, k<1$ and $m>k$, it follows that $m-k^{4}>0$. Consequently, $p_{c}-p_{a}>0$ or $p_{c}>p_{a}$.

Similarly, the difference $p_{c}-p_{b}$ can be presented as

$p_{c}-p_{b}=(1-k)\left(k^{2}+k+1\right)(k+m)(k-m)^{2}$

In this expression, $0 \leq k<1$ and $m \geq 0$, hence $1-k>$ $0, k^{2}+k+1>0, k+m>0$ and $(k-m)^{2}>0$. Consequently, $p_{c}-p_{b}>0$ or $p_{c}>p_{b}$.

Clearly, the total removed risk depends on the selection of the risk reduction options. In reality, the cost of training and the amount of reduced risk is different for each team member. In either case however, the (0-1) dynamic programming approach cannot be applied to optimise the optimal selection of risk reduction options because the amount of reduced total risk depends on the actual selection of the risk reduction options.

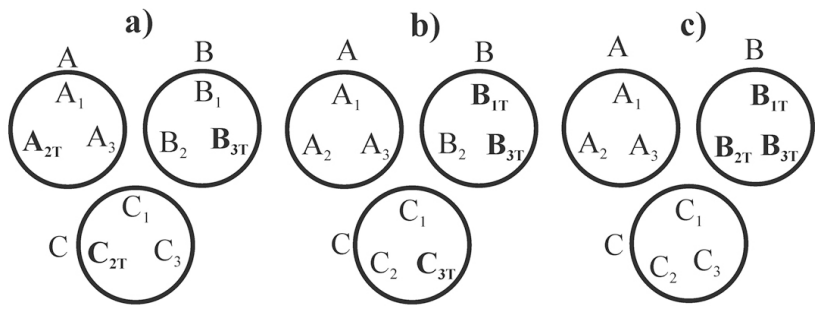

Figure 2: Three groups of people working towards eliminating the same major hazard.

Finally, consider a case of a production system similar to the one in Figure 1 which includes again old pipeline sections (O) transporting fluid from the sources $s 1, s 2$ and $s 3$ to the sink $t$.

The pipeline sections are characterised by a failure frequency (expected number of failures per year) of 8 year $^{-1}$. The capacity of each pipeline section was chosen to be 100 thousand cubic meters of fluid per day. Each failed section requires 20 days to be repaired. During the repair of a failed pipeline section, the corresponding parallel branch is not delivering any fluid.

Suppose that the available budget is used to achieve the maximum possible removed risk of lost production due to failures of pipeline sections.

An indication of the loss of production is the production unavailability $1-A$ where $A$ is the production availability of the system. Production availability is an important indicator of the performance of repairable production systems. It is 
defined as the ratio of the total amount of production fluid delivered by the system for one year in the presence of failures of the pipeline sections to the total amount of production fluid which can be potentially delivered in the absence of failures. ${ }^{[24]}$ Even a very small percentage decrease in the production availability (1\%-2\%) translates into big financial losses over the entire period of operation.

Suppose that the available risk-reduction options are: replacing 3 old pipeline sections $(\mathrm{O})$ with new sections $(\mathrm{N})$ and replacing another 3 pipeline sections with medium-age sections (M).

The failure frequency of the new sections $(\mathrm{N})$ is 0.1 year $^{-1}$ while the failure frequency of the medium-age sections (M) is 2 year $^{-1}$. The repair time for the new sections and the medium-age sections is again 20 days. Figure 3 represents different choices of pipeline sections to be replaced. a)

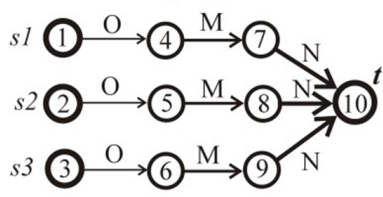

c)

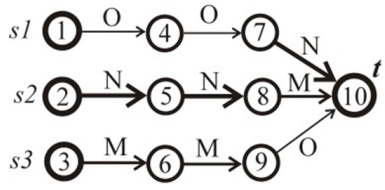

b)

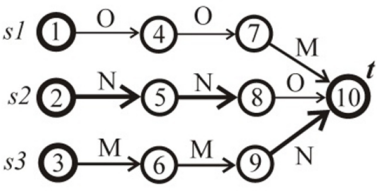

d)

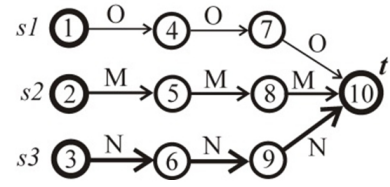

Figure 3: A production system consisting of three parallel branches and different state of deterioration of the pipeline sections.

The production availability, for one year of operation, of the different variants in Figure 3 was assessed by using the discrete-event simulator for the production availability of repairable flow networks described in Ref. 24. The production availabilities characterising the different variants were as follows: system 'a': $62.3 \%$; system 'b': $64.3 \%$; system 'c': $64.6 \%$ and system 'd': $68.4 \%$. The largest production availability was exhibited by system ' $d$ '.

The largest removed risk of lost production due to failures was achieved for variant 'd', which is associated with more than $6 \%$ increase in production availability compared to the worst variant ' $a$ '. This is a significant improvement, achieved solely by an appropriate permutation of existing interchangeable options. Rearranging interchangeable components with similar levels of reliability in separate branches, creates the very attractive opportunity to increase the reliability and availability of parallel-series systems, at no extra investment.

\subsection{An alternative formulation of the 0-1 dynamic programming approach incorporating the intrin- sic value of the available budget}

The counterexample from Table 1 exposes the danger associated with following blindly the classical (0-1) knapsack dynamic programming approach for optimising the allocation of risk reduction resources. Despite that the standard (0-1) knapsack dynamic programming algorithm always yields the exact solution in maximising the risk reduction within a fixed budget, it could still generate "solutions" wasting valuable resources on insignificant return.

The counterexample from Table 1 shows that there is clearly a need for incorporating the value of the remaining safety budget. Consequently, the requirement for a maximum total removed risk $\sum_{k \in P} r_{k}$ should be abandoned, because it leads to a wasteful use of safety resources.

This predicament can be resolved by introducing weights $(\alpha,(1-\alpha) ; 0 \leq \alpha \leq 1)$ assigned to both the amount of removed risk and the remaining budget, to reflect the value of the remaining budget. For risk reduction options all characterised by benefit/cost ratio greater than unity, what needs to be maximised is not the total amount of removed risk $\sum_{k \in P} r_{k}$ but the weighted total removed risk $\alpha \sum_{k \in P} r_{k}$ and the weighted remaining budget $(1-\alpha)\left[B-\sum_{i=1}^{n} c_{i} \times\right.$ $\left.x_{i}\right]$. This formulation prevents expending most of the remaining budget on a marginal risk reduction.

Following these considerations, the appropriate model of the optimal budget allocation among independent risk reduction options is given next:

Given the constraint:

$$
\sum_{i=1}^{n} x_{i} \times c_{i} \leq B
$$

Maximize the sum:

$$
X=\alpha \sum_{i=1}^{n} x_{i} \times r_{i}+(1-\alpha)\left[B-\sum_{i=1}^{n} c_{i} \times x_{i}\right]
$$

where $x_{i} \in\{0,1\}$ are decision variables; $x_{i}=1$, if the risk-reduction option is accepted and $x_{i}=0$, otherwise. Because the available budget $B$ is a constant, maximising the sum $X$ in equation (4) is equivalent to maximising $X=\alpha \sum_{i=1}^{n} x_{i} \times r_{i}-(1-\alpha) \sum_{i=1}^{n} c_{i} \times x_{i}$. The two summations can be combined and, as a result, what should be maximised is the expression

$$
X=\sum_{i=1}^{n} x_{i}\left[\alpha r_{i}-(1-\alpha) c_{i}\right]
$$

The weights can be conveniently altered to reflect correctly the value of unit removed risk and the value of unit remaining budget. Usually, both the removed risk and the avail- 
able budget are measured in the same monetary units and $\alpha=0.5$ is the natural weighting factor reflecting that the value of unit removed risk is the same as the value of unit remaining budget.

Thus, for ( $\alpha=0.5)$, equation (5) becomes

$$
X=0.5 \sum_{i=1}^{n} x_{i} \times\left(r_{i}-c_{i}\right)
$$

which is equivalent to maximising

$$
X=\sum_{i=1}^{n} x_{i} \times\left(r_{i}-c_{i}\right)
$$

\section{Results}

\subsection{Optimal allocation of risk reduction resources incorporating the intrinsic value of the available budget}

Implementation details related to the (0-1) knapsack dynamic programming algorithm have been discussed in detail in Ref. 23 and will not be repeated here. The difference is that the expression to be maximised is (7) instead of (1).

The (0-1) knapsack algorithm, applied to the counterexample from Table 1 now yields the correct solution. Options $A$ and $B$ are selected as optimal options and not options $C$ and $D$. This shows that the proposed model produces a superior solution compared to the standard (0-1) dynamic programming algorithm.

The proposed model also yields a solution superior to the cost-benefit solution. This will be illustrated by the next example from the railway industry. A similar example has been considered in Ref. 21. Table 2 lists 5 risk reduction measures $(A, B, C, D$ and $E$ ) associated with different amount of removed risk and different costs.

Suppose that from the central safety budget, a total budget $B=\$ 2.6$ million has been allocated to a team responsible for the reduction of platform train accidents with passengers. This is a major risk which is located in the high-risk region of the risk matrix. The first risk reduction option ' $A$ ' requires the train driver to operate a CCTV monitoring of the platform. The train will not be started if there are passengers stuck at the door, fallen onto the track or fallen between train and platform. Option $B$ requires introducing stop plungers - wall-mounted alarm devices at specified locations/intervals within the platform area which can be operated by platform staff or passengers. Trains in the platform area will be brought to a halt by operating any of these plungers. Option $C$ includes equipping the train doors with sensors to reduce the possibility of trapping and dragging passengers. Option $D$ consists of gap fillers between train and platform to reduce accidents where passengers fall between train and platform whilst boarding the train. Option $E$ includes a system preventing opening the train doors on the wrong side of the platform. ${ }^{[21]}$

The five key risk reduction options, $A, B, C, D$ and $E$ have been evaluated, and the corresponding magnitudes of removed risk and costs are according to Table 2.

Following the cost-benefit approach, the risk reduction measures $\mathrm{C}$ and $\mathrm{A}$, with the largest benefit/cost ratio will be selected. The combined cost of the selected risk reduction measures is $\$ 2.3$ million - well within the fixed budget of $\$ 2.6$ million. The removed risk is $\$ 4.7$ million.

The proposed model yields an optimal set including riskreduction options $B, C$ and $D$ with a combined cost exactly $\$ 2.6$ million (equal to the available budget) and removed risk equal to $\$ 5.1$ million. The risk-reduction ratio

$\rho=(5.1-4.7) /|2.6-2.3|=1.33$

equals $133 \%$, which indicates that the proposed solution produces a substantial return on the extra resources. The proposed model in section 2.3 yields a solution superior to the cost-benefit solution.

Table 2: Risk reduction measures with the associated costs and magnitudes of the removed risk. The total budget is $\$ 2.6$ million.

\begin{tabular}{llll}
\hline $\begin{array}{l}\text { Risk reduction } \\
\text { option }\end{array}$ & $\begin{array}{l}\text { Removed risk } \\
\text { [in millions \$] }\end{array}$ & $\begin{array}{l}\text { Cost of implement } \\
\text { [in millions \$] }\end{array}$ & $\begin{array}{l}\text { Benefit/Cost } \\
\text { ratio }\end{array}$ \\
\hline A & 2.4 & 1.2 & 2 \\
B & 1.3 & 0.7 & 1.857 \\
C & 2.3 & 1.1 & 2.09 \\
D & 1.5 & 0.8 & 1.875 \\
E & 1.6 & 0.9 & 1.777 \\
\hline
\end{tabular}

Table 3 lists 7 risk reduction options ( $A, B, C, D, E, F$ and $G)$ with removed risks and costs, according to the table. The total budget is $\mathrm{B}=\$ 170$ thousand. Following the cost-benefit approach, risk reduction options $\mathrm{A}, \mathrm{B}$ and $\mathrm{C}$, associated with the largest benefit/cost ratio will be selected. The combined cost of these risk reduction options is $T C_{C B}=\$ 140$ thousand, well within the fixed budget of $\$ 170$ thousand. The removed risk is $T R_{C B}=\$ 1478$ thousand.

Applying the model proposed earlier, yields an optimal set including risk reduction options $B, D$ and $G$. The combined cost of these options is $T C_{(0-1)}=\$ 169$ thousand (within the fixed budget of $\$ 170$ thousand) with a total removed risk $T R_{(0-1)}=\$ 1735$ thousand. The comparative ratio is

$\rho=(1735-1478) /|169-140|=8.86$

As can be verified, despite that the comparative ratio is smaller than the benefit/cost ratio of each risk-reduction option, the total risk reduction is substantial (\$257 thousand), which provides an excellent return on the invested extra budget of \$29 thousand. Clearly, the solution from the proposed 
model should be preferred to the cost-benefit solution.

Table 3: Risk reduction options with the associated costs and magnitude of the removed risk. The available budget is $\mathrm{B}=\$ 170$ thousand.

\begin{tabular}{llll}
\hline $\begin{array}{l}\text { Risk reduction } \\
\text { option }\end{array}$ & $\begin{array}{l}\text { Removed risk } \\
\text { [in thousands \$] }\end{array}$ & $\begin{array}{l}\text { Cost of implement. } \\
\text { [in thousands \$] }\end{array}$ & $\begin{array}{l}\text { Benefit/Cost } \\
\text { ratio }\end{array}$ \\
\hline A & 442 & 41 & 10.78 \\
B & 525 & 50 & 10.5 \\
C & 511 & 49 & 10.4 \\
D & 593 & 59 & 10.05 \\
E & 546 & 55 & 9.927 \\
F & 564 & 57 & 9.89 \\
G & 617 & 60 & 10.28 \\
\hline
\end{tabular}

Table 4 lists 24 different risk-reduction options and available budget $\mathrm{B}=\$ 6404180$. The options selected in the optimal set by the proposed model are shown in the last column of the table.

Table 4: Risk-reduction options with the associated costs and magnitude of the removed risk. The total budget is $\$ 6404180$.

\begin{tabular}{|c|c|c|c|c|}
\hline $\begin{array}{l}\text { Risk reduction } \\
\text { option }\end{array}$ & $\begin{array}{l}\text { Removed } \\
\text { risk, \$ }\end{array}$ & $\begin{array}{l}\text { Cost of imple- } \\
\text { ment.\$ }\end{array}$ & $\begin{array}{l}\text { Benefit/Cost } \\
\text { ratio }\end{array}$ & $\begin{array}{l}\text { Selection } \\
\text { indicator }\end{array}$ \\
\hline 1 & 825594 & 382745 & 2.1570 & 1 \\
\hline 2 & 1677009 & 799601 & 2.0973 & 1 \\
\hline 3 & 1676628 & 909247 & 1.8440 & 0 \\
\hline 4 & 1523970 & 729069 & 2.0903 & 1 \\
\hline 5 & 943972 & 467902 & 2.0175 & 1 \\
\hline 6 & 97426 & 44328 & 2.1978 & 1 \\
\hline 7 & 69666 & 34610 & 2.0129 & 0 \\
\hline 8 & 1296457 & 698150 & 1.8570 & 0 \\
\hline 9 & 1679693 & 823460 & 2.0398 & 0 \\
\hline 10 & 1902996 & 903959 & 2.1052 & 1 \\
\hline 11 & 1844992 & 853665 & 2.1613 & 1 \\
\hline 12 & 1049289 & 551830 & 1.9015 & 0 \\
\hline 13 & 1252836 & 610856 & 2.0510 & 1 \\
\hline 14 & 1319836 & 670702 & 1.9678 & 0 \\
\hline 15 & 953277 & 488960 & 1.9496 & 0 \\
\hline 16 & 2067538 & 951111 & 2.1738 & 1 \\
\hline 17 & 675367 & 323046 & 2.0906 & 0 \\
\hline 18 & 853655 & 446298 & 1.9127 & 0 \\
\hline 19 & 1826027 & 931161 & 1.9610 & 0 \\
\hline 20 & 65731 & 31385 & 2.0943 & 0 \\
\hline 21 & 901489 & 496951 & 1.8140 & 0 \\
\hline 22 & 577243 & 264724 & 2.1805 & 1 \\
\hline 23 & 466257 & 224916 & 2.0730 & 1 \\
\hline 24 & 369261 & 169684 & 2.1762 & 1 \\
\hline
\end{tabular}

For a small number of risk reduction options (up to 12), the proposed model has been validated by using a recursive backtracking algorithm generating, evaluating and comparing all possible combinations of risk-reduction options, after which the best combination, associated with the largest sum $X=\sum_{i=1}^{n} x_{i} \times\left(r_{i}-c_{i}\right)$ is selected.

A validation test has been conducted including 12 risk reduction options, with removed risks and costs according to Table 5. The optimal selection produced by the proposed model is given in the last column of Table 5. The execu- tion of the recursive backtracking algorithm yielded options $2,3,4,6,9,10,11$ as optimal options, with total cost $\$ 1577$ thousand. This result matched exactly the result from the proposed model in section 2.2.

Table 5: A validation test example including 12 risk-reduction options, the associated costs and magnitudes of the removed risk. The total budget is $\$ 1600$ thousand.

\begin{tabular}{lllll}
\hline $\begin{array}{l}\text { Risk reduction } \\
\text { option }\end{array}$ & $\begin{array}{l}\text { Removed risk } \\
{[\mathbf{x} \text { 1000 \$] }}\end{array}$ & $\begin{array}{l}\text { Cost of option } \\
{[\mathbf{x} \text { 1000 \$] }}\end{array}$ & $\begin{array}{l}\text { Benefit/Cost } \\
\text { ratio }\end{array}$ & $\begin{array}{l}\text { Selection } \\
\text { indicator }\end{array}$ \\
\hline 1 & 245 & 182 & 1.35 & 0 \\
2 & 311 & 166 & 1.87 & 1 \\
3 & 412 & 240 & 1.72 & 1 \\
4 & 567 & 378 & 1.5 & 1 \\
5 & 188 & 112 & 1.68 & 0 \\
6 & 443 & 277 & 1.6 & 1 \\
7 & 116 & 79 & 1.47 & 0 \\
8 & 89 & 45 & 1.98 & 0 \\
9 & 398 & 217 & 1.83 & 1 \\
10 & 178 & 98 & 1.82 & 1 \\
11 & 477 & 201 & 2.37 & 1 \\
12 & 289 & 245 & 1.18 & 0 \\
\hline
\end{tabular}

A number of additional tests have also been conducted, with a different number of risk-reduction options. Invariably, the results from the recursive backtracking procedure matched exactly the results from the proposed model. The worst-case running time of the (0-1) dynamic programming algorithm for optimal allocation of a safety budget is $O(n \times B)$ where, $n$ is the number of available options and $B$ is the size of the budget as an integer number. Expressing the available budget $B$, the removed risk and the cost of the options as numbers (e.g. rounded to the nearest thousand) makes the (0-1) dynamic programming algorithm very efficient, which is indicated by the results for the set of options in Table 4. Despite the large budget and the presence of risk-reduction options with very different costs, the solution was obtained by the (0-1) knapsack algorithm after $1.75 \mathrm{~s}$, on a computer with a processor Intel (R) Core (TM) 2 Duo CPU T9900 @ $3.06 \mathrm{GHz}$.

Going back to the second limitation of the classical (0-1) dynamic programming approach, for two sets including the same number of identical independent risk-reduction options, because of synergistic effects, some sets of options remove more risk compared to other sets of options. The classical (0-1) knapsack dynamic programming approach cannot handle cases where synergistic effects are present.

To be applicable to problems of optimal risk reduction, the (0-1) knapsack dynamic programming approach must operate only with risk-reduction options for which no synergistic effects are present and the total removed risk depends only on the sum of the removed risks by the separate options. The risk-reduction options in various industries possess this property. Examples of such options have already been given from the railway industry.

A good test signalling the presence of synergistic effects among the risk reduction options is to assume temporarily 
that each of the risk reduction options has the same cost and removes the same amount of risk. Next, a check is performed whether any selection of $m$ risk reduction options removes the same amount of total risk, where $m$ is any feasible number of selected options. If particular selections of options remove more total risk than other selections, synergistic effects are present and the (0-1) dynamic programming approach cannot be applied.

Consider a case where again, the available risk reduction options are investments in training people. In the first case, three teams $A, B$ and $C$, each of which contains three independently working team members, work in three different safety areas. Team members $A_{1}, A_{2}$ and $A_{3}$ reduce the risk of derailment; team members $B_{1}, B_{2}$ and $B_{3}$ reduce the risk of train collision and team members $C_{1}, C_{2}$ and $C_{3}$ reduce the risk of train platform accidents. In this case, no requirement is present for each team member to be successful in their allocated task for the risk to be reduced. The effort of each team member reduces independently the corresponding risk. Investing in training of team members results in a proportional reduction of the corresponding risk.

Now assume that the efforts from each team member reduce the same amount of risk and investment in training of any team member results in the same amount of removed extra risk. If the efforts of each team member result in the same amount of removed risk, it is clear that selecting any combination of $\mathrm{m}$ options (people to train) will result in the same amount of reduced total risk. No synergistic effect is present and the total removed risk does not depend on the selection of the risk reduction options. In reality, the cost of training and the amount of reduced risk will be different for each person and the (0-1) dynamic programming approach can be applied to determine the optimal selection of people for training.

This case can be compared with the case already discussed in Section 2.2 where the three teams work in parallel on the task of eliminating the same major hazard and the total risk is reduced if at least one of the teams succeeds in eliminating the hazard. Again, it can be assumed temporarily that the efforts of each team member have the same impact. From the analysis presented in Section 2.2 however, it is clear that despite that all risk reduction options are identical and independent, because of the synergistic effect, the (0-1) dynamic programming approach cannot be applied to optimise this case.

In many real cases, the risk-reduction options are not statistically dependent but this does not preclude the use of the (0-1) knapsack problem for determining the optimal resource allocation. In some cases, the selection of one of the risk-reduction options $A_{i}$ automatically excludes the selection of a series of other options $A_{j}, i \neq j\left(A_{i} \cap A_{j}=\varnothing\right)$. For example, purchasing a particular type $A_{i}$ signalling system/breaks excludes purchasing another type $A_{j}$ of sig- nalling system/breaks. In other cases, the selection of a risk-reduction option $A$ requires the selection of other riskreduction options $B_{i}\left(A \rightarrow B_{i}\right)$. Such is the case where purchasing a signalling system (option $A$ ) requires investing in training (option $B_{1}$ ) and purchasing an improved communication system (option $B_{2}$ ).

There are also asymmetrical risk-reduction options where option $A$ for example can be selected without option $B$ but option $B$ cannot be selected without option $A$. For example, option $A$ consisting of purchasing calibration and maintenance services for equipment controlling an important functional parameter (e.g. temperature, pressure, concentration) cannot be done without purchasing the controlling equipment.

These numerous option constraints can be presented as additional constraints to the (0-1) knapsack problem. The optimal solution can still be found by solving the (0-1) knapsack problem provided that no synergistic effects are present for the different sets of options.

\subsection{Optimal strategy in reducing the total risk in parallel-series systems}

The parallel-series logical arrangement example in section 2.2 demonstrated that the (0-1) dynamic programming approach cannot be used if synergistic effects are present. The parallel-series logical arrangement is a common logical arrangement in safety devices working in parallel for preventing the occurrence of a particular risk event. Consider a safety-critical system for detecting the release of heath from incipient fire, based on $n$ heath detectors working in parallel, each of which consist of a heath sensor, control block and an alarm. Upon fire, the system detects the heath release if at least one of the detectors working in parallel detects the heath release. This system has a parallel-series logical arrangement because the heath detectors are logically arranged in parallel while the components building them (heath sensor, control block and an alarm) are logically arranged in series.

Because systems with parallel-series logical arrangements (see Figure 4) are very common, and the optimal selection of risk reduction options cannot be resolved by the (0-1) dynamic programming approach, a specific method should be developed for accomplishing the task of optimal resources allocation which delivers the smallest risk of system failure.

The cases in Figure 1, Figure 2c and Figure 3d are examples of well-ordered parallel-series systems. A well-ordered parallel-series arrangement is obtained if the available components are used to build the branch with the highest possible reliability/availability, the remaining components are used to build the next parallel branch with the highest possible reliability/availability and so on, until the entire parallel- 
series arrangement is built.

Another example of a well-ordered parallel-series system is the system in Figure 4, where in the parallel branches there are three pre-existing components with reliabilities $r_{01}=0.78, r_{02}=0.75, r_{03}=0.80$. These components are always attached to the corresponding branches and cannot be swapped. There are also empty sockets which can accommodate type-A components and type-B components. There are two type-A components: an old component with reliability 0.57 and a medium-age component with reliability 0.67 . There are also six type-B components: Four old components with reliability 0.7 and two medium-age components with reliability 0.8 . The system with the highest possible reliability (the largest removed risk of system failure) is the system shown in Figure 4. In this system, the branch with the highest possible reliability (the second branch) cannot be improved by interchanging type- $B$ components with type- $B$ components from other branches. The next highest-reliability branch (the third branch) cannot be improved by interchanging components with the less reliable first branch.

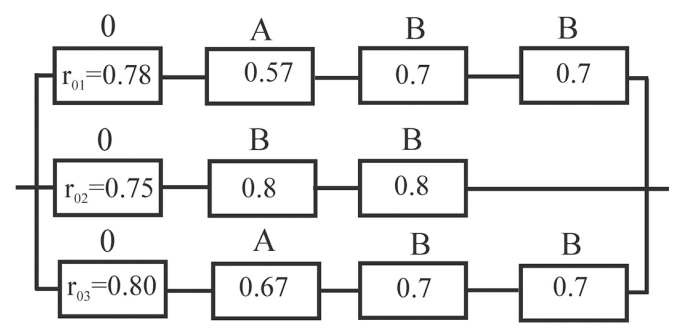

Figure 4: A parallel-series logical arrangement with three pre-existing components with specified reliabilities and eight interchangeable components.

Yet another example of a parallel-series mechanical system is the one in Figure 5 featuring a pipeline with three interchangeable valves V1, V2 and V3 of the same type. The valves are initially open and are closed on demand by the actuators A1, A2 and A3 (all of the same type and therefore interchangeable), which are energized by the control modules CM1,CM2 and CM3, also of the same type and therefore interchangeable.

The reliability network of the system from Figure $5 \mathrm{a}$ is given in Figure 5b. With respect to the function stopping the fluid in the pipeline on demand, the valves are logically arranged in parallel, while the actuators and the control modules are logically arranged in series (see Figure 5b). This is because at least one of the three valve blocks needs to be operational to stop the fluid in the pipeline. For a valve block to be operational, the valve, the corresponding actuator and control module must all be operational.

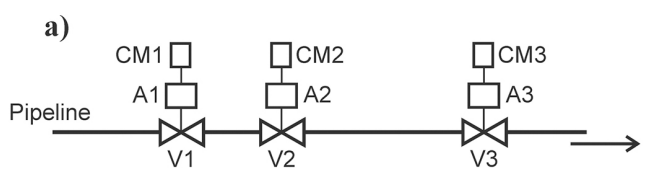

b)

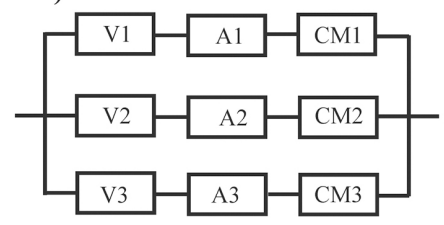

Figure 5: (a) A functional diagram of three valve blocks on a pipeline; (b) Reliability block diagram of the function "stopping the fluid in the pipeline".

The results obtained for a number of well-ordered parallelseries systems have been verified by a computer simulation. The computer simulation consisted of specifying the reliabilities of the interchangeable components in the branches and calculating the reliability/availability of the well-ordered system. The second phase of the validation program was a "random shuffle" of the interchangeable components in the branches, by generating random indices of components from different branches and swapping their reliability values. The swapping guarantees that any resultant system includes exactly the same set of interchangeable components as the initial system. After the random shuffle, the reliability/availability of the shuffled system was calculated and compared with the reliability of the well-ordered system. If the reliability/availability of the well-ordered system was greater than or equal to the reliability of the shuffled system, the content of a counter was increased. At the end, the probability was calculated that the well-ordered system has reliability/availability not smaller than the reliability of the shuffled system. In all conducted simulations, this probability was always equal to one, which leads to the conjecture that the well-ordered parallel-series arrangements are characterised by the largest reliability/availability. These results lead to establishing the following general result:

Theorem. Among all possible parallel-series logical arrangements, the well-ordered parallel-series logical arrangement possesses the highest possible reliability.

Proof. This proposition will be proved by contradiction and by making use of the extreme principle. Suppose that there is a parallel-series logical arrangement which is not well-ordered and which possesses the highest possible reliability. Without loss of generality, suppose that the parallel branches in this arrangement have been re-arranged in such a way that for any two branches ' $i^{\prime},{ }^{\prime} j^{\prime}$ for which $i<j$, the reliability $R_{i}$ of the branch ' $i$ ' is not less than the $R_{j}$ reliability of branch ' $j^{\prime}\left(R_{i} \geq R_{j}\right)$. If the parallel-series arrangement is not well-ordered, then there must be two branches with indices $a$ and $b(a<b)$ and reliabilities $R_{a} \geq R_{b}$, where there will be at least one component in branch $a$ 
with a smaller reliability than the reliability of the analogous component in branch $b$. Suppose that $R_{a}=a_{1} a_{2} \times \cdots \times a_{n a}$ and $R_{b}=b_{1} b_{2} \times \cdots \times b_{n b}$ are the reliabilities of branches $a$ and $b$ and $n a, n b, n b$ are the number of components in branches $a$ and $b$, correspondingly. The quantities $a_{i}$ and $b_{i}$ stand for the reliabilities of the components in branches 'a' and 'b', respectively. Without loss of generality, suppose that ' $n a^{\prime}$ and ' $n b^{\prime}$ are the indices of the two analogous components in branches 'a' and 'b', for which $a_{n a}<b_{n b}$ is fulfilled.

The reliability of the initial system can be presented as

$$
R_{\text {sys } 1}=1-\left(1-a_{1} a_{2} \times \cdots \times a_{n a}\right)\left(1-b_{1} b_{2} \times \cdots \times b_{n b}\right) \times\left[1-R_{\text {rest }}\right]
$$

where $R_{\text {rest }}$ is the reliability of the rest of the parallel-series arrangement (not including branches ' $a$ ' and ' $b$ ').
After swapping the components with indices $n a$ and $n b$, the reliability of the resultant system becomes

$$
R_{\text {sys } 2}=1-\left(1-a_{1} a_{2} \times \cdots \times a_{n a-1} b_{n b}\right)\left(1-b_{1} b_{2} \times \cdots \times b_{n b-1} a_{n a}\right) \times\left[1-R_{r e s t}\right]
$$

Subtracting (9) from (8) yields:

$$
R_{s y s 1}-R_{s y s 2}=\left(a_{n a}-b_{n b}\right)\left(a_{1} a_{2} \times \cdots \times a_{n a-1}-b_{1} b_{2} \times \cdots \times b_{n b-1}\right) \times\left[1-R_{r e s t}\right]
$$

Because $R_{a}=a_{1} a_{2} \times \cdots \times a_{n a} \geq R_{b}=b_{1} b_{2} \times \cdots \times b_{n b}$ (by the way the branches have been arranged in descending order according to their reliability) and because $a_{n a}<b_{n b}$ by assumption, the inequality

$$
a_{1} a_{2} \times \cdots \times a_{n a-1}>b_{1} b_{2} \times \cdots \times b_{n b-1}
$$

holds, which means that in equation (10),

$a_{1} a_{2} \times \cdots \times a_{n a-1}-b_{1} b_{2} \times \cdots \times b_{n b-1}>0$

Since $1-R_{\text {rest }}>0$ and $a_{n a}-b_{n b}<0$, the right hand side of equation (10) is negative, which means that the resultant system (after the swapping of components) has a higher reliability $\left(R_{s y s 2}>R_{s y s 1}\right)$. This contradicts the assumption that the initial system (before the swap of components) was the system with the highest possible reliability. Therefore, the reliability of a system which is not well-ordered, can always be improved by swapping components between parallel branches until a well-ordered system is finally attained. The well-ordered system is unique and there can be no distinct two well-ordered systems. Because a parallel-series system can either be a well-ordered or not well-ordered system, the well-ordered system has a higher reliability compared to any other arrangement. The theorem has been proved.

This result provides an opportunity to remove the maximum amount of total risk of failure in parallel-series arrangements by concentrating the available resources on renewing single parallel branches as opposed to renewing randomly selected components in the system.

This result also provides the valuable opportunity to improve the reliability/availability of common engineering systems with parallel-series logical arrangement of their components without the knowledge of their reliabilities and without any investment. Unlike traditional approaches, which invariably require resources to achieve a reliability improvement and system risk reduction, a risk reduction can also be achieved at no extra cost, by appropriate permutation of the available interchangeable components between the parallel branches.

Components of similar level of deterioration (reliability levels) should be placed in the same parallel branch. Although this study is related to systems with parallel-series logical arrangement, it clearly identifies the need to explore possibilities for improving the reliability and availability of systems with topologically complex arrangement, at no extra cost, achieved solely by permutations of interchangeable components.

\section{Conclsuions}

1) The classical (0-1) knapsack dynamic programming formulation used for optimal allocation of safety resources to achieve a maximum benefit yields highly undesirable solutions, wasting resources on insignificant risk reduction.

2) The classical knapsack dynamic programming approach maximises the total benefit derived from items 'filling a space' with no intrinsic value. While this is an appropriate formulation for packing and cargo loading problems, for applications involving capital budgeting, this formulation is deeply flawed. The reason is that budgets do have intrinsic value and their efficient use is just as important as the maximisation of the benefit derived from the budget allocation. 
3) A new resource allocation model has been proposed, where the weighted sum of the benefit (total removed risk) and the remaining budget is maximised. The proposed model produces solutions superior to both - the classical (0-1) dynamic programming approach and the cost-benefit approach.

4) The proposed approach promotes a more efficient use of the risk reduction resources by preventing them from being locked into insignificant benefit improvement. The proposed approach permits freeing risk-reduction resources from areas of diminishing returns to other areas where more risk could be removed with the same levels of investment.

5) Because of synergistic effects, sets including the same number of identical options could remove different amount of total risk. The existence of synergistic effects does not permit the application of the (0-1) dynamic programming approach. In this case, specific methods for optimal resource allocation should be developed.

\section{References}

[1] Genserik L.L.R., K. Sorensen, Optimal allocation of safety and security resources. Chemical engineering transactions. 2013; 31: 397 402 .

[2] Weingartner H. and D. Ness. Methods for the solution of the multidimensional 0/1 knapsack problem. Operations Research. 1967; 15(1): 83-103. http://dx.doi.org/10.1287/opre.15.1.83

[3] Horowitz, E., Sahni, S. Computing partitions with applications to the Knapsack Problem. Journal of the ACM. 1974; 21: 277-292.

[4] Moser M., D. Jokanovic, and N. Shiratori. An algorithm for the multi-dimensional multiple-choice knapsack problem. IEICE Transactions on Fundamentals of Electronics, Communications and Computer Sciences. 1997; 80(3): 582-589.

[5] Martello S., Toth P. Knapsack problems: Algorithms and computer implementations. John Willey \& Sons. 1990.

[6] Martello, S., Pisinger, D., Toth, P. New Trend in exact algorithms for the 0-1 knapsack problem. European Journal of Operational Research. 2000; 123: 325-332. http://dx.doi.org/10.1016/ S0377-2217(99) 00260-X

[7] Beier R., B.Vocking. Probabilistic analysis of knapsack core algorithms, SODA '04 Proceedings of the fifteenth annual ACM-SIAM symposium on Discrete algorithms. Society for Industrial and Applied Mathematics, Philadelphia, PA, USA. 2004: 468-477.

[8] Fréville A. The multidimensional 0-1 knapsack problem: An overview. European Journal of Operational Research. 2004; 155 (1): 1-21. http://dx.doi.org/10.1016/S0377-2217(03)00274-1

[9] Šeda M. Solving Resource-Constrained Project Scheduling Problem As a Sequence of Multi-Knapsack Problems. WSEAS Transactions on Information Science and Applications. 2006; 3(10): 1785-1791.

[10] Chandra A., N.M. Menon and B.K. Mishra. Budgeting for Information Technology. International Journal of Accounting Information Systems. 2007; 8(4): 264-282.

[11] Dasgupta, S., Papadimitriou, C., Vazirani, U. Algorithms. McGraw Hill, Boston. 2008.
6) For systems with parallel-series logical arrangement, the maximum amount of risk is removed by using the available resources preferentially, on improving the reliability of operations/components building an entire parallel branch. Improving the reliability of randomly selected operations/components leads to a sub-optimal risk reduction.

7) The concept 'well-ordered parallel-series arrangement' has been introduced and, by using the extreme principle, a relevant theorem has been stated and proved: 'among all possible parallel-series logical arrangements, the wellordered parallel-series arrangement has the highest reliability'.

8) The proposed approach, based on well-ordered parallelseries arrangement, makes it possible to increase the reliability and availability of engineering systems at no extra investment. This creates the attractive opportunity to significantly increase the competitiveness of many engineering companies.
[12] Boyer V., D. El Baz, Moussa Elkihel. Solution of multidimensional knapsack problems via cooperation of dynamic programming and branch and bound. European J. Industrial Engineering. 2010: 4(4).

[13] Puchinger J., G.R. Raidl, U. Pferschy, The Multidimensional Knapsack Problem: Structure and Algorithms. INFORMS Journal on Computing. 2010; 22 (2): 250-265. http://dx.doi.org/10. 1287 /ijoc.1090.0344

[14] Lin G., W. Zhu and M.M.Ali. An exact algorithm for the 0-1 linear knapsack problem with a single continuous variable. Journal of Global Optimization. 2011; 50(4): 657-673. http://dx.doi . org/10.1007/s10898-010-9642-5

[15] Varnamkhasti M.J. Overview of the Algorithms for Solving the Multidimensional Knapsack Problems. Advanced Studies in Biology. 2012; 4(1): 37-47.

[16] Lalami M.E., M. Elkihel, D. El Baz and V. Boyer. A procedurebased heuristic for 0-1 Multiple Knapsack Problems. Int. J. Mathematics in Operational Research. 2012; 4(3): 214-224. http://dx. doi.org/10.1504/IJMOR . 2012.046684

[17] Bellman R. Dynamic programming. Princeton, N. J.: Princeton University Press. 1957.

[18] Richter A., Brandeau M.L. and Owens D.K. An Analysis of Optimal Resource Allocation for Prevention of Infection with Human Immunodeficiency Virus (HIV) in Injection Drug Users and NonUsers. Medical Decision Making. 1999; 19(2): 167-179.

[19] Todinov M.T. New models for optimal reduction of technical risks. Engineering Optimization. 2012; 45 (6): 719-743.

[20] Mehr A.F. and Tumer. I.Y. Risk-based decision-making for managing resources during the design of complex space exploration systems. J. Mech. Design. 2006; 128: 1014-1022.

[21] Weli E., M.Todinov. A new approach to risk reduction in the railway industry. Infrastructure Risk and Resilience: Transportation, 2013. pp. 47-52, The Institution of Engineering and Technology.

[22] Pigman J.G., Agent K.R., Mayes, J.G. and C.V.Zeger. Optimal highway safety improvement investments by dynamic programming. Research report. 1974: 412.

[23] Todinov M.T., E.Weli. Optimal risk reduction in the railway industry, by using dynamic programming, WASET. 2013; 79: 220-224.

[24] Todinov M.T., Flow networks, Elsevier, 2013. 